\title{
CpG island density and its correlations with genomic features in mammalian genomes Leng $\mathrm{Han}^{*+*}$, Bing $\mathrm{Su}^{\dagger}$, Wen-Hsiung Liףl and Zhongming $\mathrm{Zhao}^{*} \mathrm{Y}$
}

Addresses: *Department of Psychiatry, Virginia Commonwealth University, Richmond, VA 23298, USA. 'State Key Laboratory of Genetic Resources and Evolution, Kunming Institute of Zoology, Chinese Academy of Sciences, Kunming, Yunnan 650223, China. ${ }^{\ddagger}$ Graduate School, Chinese Academy of Sciences, Beijing 100039, China. \$Kunming Primate Research Center, Chinese Academy of Sciences, Kunming, Yunnan 650223, China. `Department of Ecology and Evolution, University of Chicago, Chicago, IL 60637, USA. ¥Department of Human Genetics and Center for the Study of Biological Complexity, Virginia Commonwealth University, Richmond, VA 23284, USA.

Correspondence: Zhongming Zhao. Email: zzhao@vcu.edu

Published: 13 May 2008

Genome Biology 2008, 9:R79 (doi:10.1 186/gb-2008-9-5-r79)

The electronic version of this article is the complete one and can be found online at http://genomebiology.com/2008/9/5/R79
Received: 7 April 2008

Revised: 8 April 2008

Accepted: 13 May 2008

(c) 2008 Han et al.; licensee BioMed Central Ltd.

This is an open access article distributed under the terms of the Creative Commons Attribution License (http://creativecommons.org/licenses/by/2.0), which permits unrestricted use, distribution, and reproduction in any medium, provided the original work is properly cited.

\begin{abstract}
Background: $C_{P G}$ islands, which are clusters of $C_{P G}$ dinucleotides in GC-rich regions, are considered gene markers and represent an important feature of mammalian genomes. Previous studies of CPG islands have largely been on specific loci or within one genome. To date, there seems to be no comparative analysis of CPG islands and their density at the DNA sequence level among mammalian genomes and of their correlations with other genome features.
\end{abstract}

Results: In this study, we performed a systematic analysis of $C_{p} G$ islands in ten mammalian genomes. We found that both the number of $C_{P} G$ islands and their density vary greatly among genomes, though many of these genomes encode similar numbers of genes. We observed significant correlations between CPG island density and genomic features such as number of chromosomes, chromosome size, and recombination rate. We also observed a trend of higher $C_{P G}$ island density in telomeric regions. Furthermore, we evaluated the performance of three computational algorithms for $C_{p} G$ island identifications. Finally, we compared our observations in mammals to other non-mammal vertebrates.

Conclusion: Our study revealed that $C_{p} G$ islands vary greatly among mammalian genomes. Some factors such as recombination rate and chromosome size might have influenced the evolution of $C_{P G}$ islands in the course of mammalian evolution. Our results suggest a scenario in which an increase in chromosome number increases the rate of recombination, which in turn elevates GC content to help prevent loss of $C_{P} G$ islands and maintain their density. These findings should be useful for studying mammalian genomes, the role of $C_{p G}$ islands in gene function, and molecular evolution. 


\section{Background}

CpG islands (CGIs) are clusters of $\mathrm{CpG}$ dinucleotides in GCrich regions and represent an important feature of mammalian genomes [1]. Mammalian genomic DNA generally shows a great deficit of $\mathrm{CpG}$ dinucleotides, for example, the ratio of the observed over the expected CpGs $\left(\mathrm{Obs}_{\mathrm{CpG}} / \operatorname{Exp}_{\mathrm{CpG}}\right)$ is approximately $0.20-0.25$ in the human and mouse genomes [2-4]. This deficit is largely attributed to the hypermutability of methylated CpGs to TpGs (or CpAs in the complementary strand) [5,6]. In comparison, CpGs in CGIs are often unmethylated and their frequencies are close to random expectation (for example, $\mathrm{Obs}_{\mathrm{CpG}} / \operatorname{Exp}_{\mathrm{CpG}}=\sim 0.8$ in the promoter-associated CGIs [7]). CGIs are often associated with the 5 ' end of genes and considered as gene markers [8,9]. However, a comparison of the human, mouse, and rat genomes indicated that, although these three genomes encode similar numbers of genes, the number of CGIs in the mouse (15,500) or rat $(15,975)$ genome is far fewer than that $(27,000)$ identified in the non-repetitive portions of the human genome [10-12]. The difference is probably due to a faster rate of loss of CGIs in the rodent lineage, rather than faster gains of CGIs in the human lineage [7,9]. However, it remains unclear whether the loss-of-CGI model holds for other mammalian genomes. Furthermore, to our best knowledge, there has been no comprehensive analysis of CGIs and their density at the DNA sequence level in mammals.

There are three major algorithms for identifying CGIs in a genomic sequence. The original algorithm was proposed by Gardiner-Garden and Frommer [13] in 1987; the three parameters are GC content $>50 \%, \mathrm{Obs}_{\mathrm{CpG}} / \operatorname{Exp}_{\mathrm{CpG}}>0.60$, and length $>200 \mathrm{bp}$. This algorithm, often with some modifications, has been widely applied in the analysis of CGIs in single genes, small sets of genomic sequences, or single genomes. However, many repeats (for example, Alu), which are abundant in the vertebrate genome, also meet the criteria, so this algorithm has usually been used to scan CGIs only in nonrepeat portions of the genome $[2,11,12]$. Second, Takai and Jones [14] evaluated the three parameters in Gardiner-Garden and Frommer's algorithm using human gene data and suggested an optimal set of parameters (GC content $\geq 55 \%$, $\mathrm{Obs}_{\mathrm{CpG}} / \operatorname{Exp}_{\mathrm{CpG}} \geq 0.65$, and length $\geq 500 \mathrm{bp}$ ). This algorithm can effectively exclude false positive CGIs from repeats and more likely identify CGIs associate with the 5 ' end of human genes; it seems to be suitable for other genomes too [14]. Third, more recently, Hackenberg et al. [15] developed a new algorithm, namely CpGcluster, that entirely depends on the statistical significance of a $\mathrm{CpG}$ cluster from random sequences in the same chromosome. Because CpGcluster does not require a minimum length (for example, it identified CpG clusters as short as $8 \mathrm{bp}$ ) [15], it likely identifies many more CGIs (for example, 197,727 in the human genome) than other algorithms. In particular, CpGcluster may exaggerate the number of CGIs (that is, CpG clusters) in low GC-content chromosomes, which often have low gene density, because its CpG clusters were identified relative to the background (ran- dom) CpG property. Another similar CpG cluster algorithm identifies $\mathrm{CpG}$ clusters by requiring a minimum number of CpGs in each sequence fragment [16]. Since loss of CGIs is likely an evolutionary trend in at least some genomes [7,9,17], CpGcluster may be able to identify those CGIs that have undergone degradation and thus can not meet the criteria of Takai and Jones' or Gardiner-Garden and Frommer's algorithms.

Our major aim is to survey extant CGIs (that is, CGIs that meet the three typical criteria: length, GC content, and $\mathrm{Obs}_{\mathrm{CpG}} / \operatorname{Exp}_{\mathrm{CpG}}$ ) and their distribution in today's genomes, rather than to identify regions that might originally be CGIs, even though they do not meet the three typical criteria. A comparative study of the features of such CGIs will be helpful for studying the evolution of CGIs and sequence composition changes in the course of genome evolution. Recent genome sequencing projects have released a number of mammalian genomes with good quality annotations, but only few nonmammalian vertebrate genomes. Thus, in this study we focused on the analysis and comparison of CGIs and their correlations with genomic features in mammalian genomes. For our aim, it is appropriate to apply the same CGI detection algorithm to screen CGIs in multiple genomes for comparison. According to the introduction of the three algorithms above, we selected Takai and Jones' algorithm as a major algorithm in this study.

We conducted a systematic survey of CGIs in ten sequenced mammalian genomes: eight completely sequenced eutherian genomes (human (Homo sapiens), chimpanzee (Pan troglodytes), macaque (Macaca mulatta), mouse (Mus musculus), rat (Rattus norvegicus), dog (Canis familiaris), cow (Bos taurus), and horse (Equus caballus)); one completely sequenced metatherian genome (opossum (Monodelphis domestica)); and one prototherian genome (platypus (Ornithorhynchus anatinus)) whose sequence was completed with a $6 \times$ coverage, though it has not been completely assembled. We also compared the observations from these mammals to seven other non-mammal vertebrates.

\section{Results \\ CGIs and CGI density in ten mammalian genomes}

We first present our analysis of CGIs identified by Takai and Jones' algorithm [14] in ten mammalian genome sequences. The conclusions are essentially the same when we used the popular algorithm by Gardiner-Garden and Frommer [13] or the recently developed algorithm CpGcluster [15] (see Discussion). The species names and the sources of genome sequences are shown in the Materials and methods. Table 1 summarizes the genome information and statistics of CGIs. Except for the platypus, these genomes had similar sizes (2.0$3.3 \mathrm{~Gb})$ and similar numbers of annotated genes (20,00o30,000; Additional data file 1). However, both the number of CGIs and the CGI density (measured by the average number 
Table I

\begin{tabular}{|c|c|c|c|c|c|c|c|c|c|c|}
\hline \multirow[b]{2}{*}{ Species } & \multicolumn{5}{|c|}{ Genome } & \multicolumn{5}{|c|}{$C_{p} G$ islands } \\
\hline & Size $(\mathrm{Gb})^{*}$ & $\begin{array}{l}\text { Number of } \\
\text { chromosome } \\
\text { pairs }\end{array}$ & $\begin{array}{c}\text { Number of } \\
\text { arms }{ }^{\dagger}\end{array}$ & $\begin{array}{c}\mathrm{GC} \\
\text { content } \\
(\%)\end{array}$ & $\begin{array}{l}\text { Obs }_{C_{p G}} l \\
\operatorname{Exp}_{C_{p G}}\end{array}$ & $\begin{array}{c}\text { Number of } \\
\text { CGls }\end{array}$ & $\begin{array}{c}\text { CGI } \\
\text { density } \\
(/ \mathrm{Mb})\end{array}$ & $\begin{array}{l}\text { Avgerage } \\
\text { length (bp) }\end{array}$ & $\begin{array}{c}\text { GC } \\
\text { content } \\
(\%)\end{array}$ & $\begin{array}{l}\text { Obs }_{C_{p G}} l \\
\operatorname{ExP}_{C_{p G}}\end{array}$ \\
\hline Human & 2.85 & 23 & 82 & 40.9 & 0.236 & 37,531 & 13.2 & 1,089 & 62.0 & 0.743 \\
\hline Chimpanzee & 2.75 & 24 & 84 & 40.7 & 0.233 & 35,845 & 13.0 & $|, 01|$ & 60.3 & 0.761 \\
\hline Macaque & 2.65 & 21 & 84 & 40.7 & 0.245 & 39,498 & 14.9 & 957 & 60.8 & 0.749 \\
\hline Mouse & 2.48 & 20 & 40 & 41.7 & 0.192 & 20,458 & 8.2 & 1,043 & 60.6 & 0.756 \\
\hline Rat & 2.48 & 21 & 64 & 41.9 & 0.220 & 19,568 & 7.9 & 1,004 & 59.7 & 0.758 \\
\hline Dog & 2.31 & 39 & 80 & 41.0 & 0.244 & 58,327 & 25.3 & 1,102 & 62.2 & 0.753 \\
\hline Cow & 2.29 & 30 & 62 & 41.9 & 0.236 & 36,729 & 16.0 & 1,023 & 61.2 & 0.740 \\
\hline Horse & 2.03 & 32 & 92 & 41.0 & 0.285 & 33,135 & 16.3 & 937 & 59.2 & 0.749 \\
\hline Opossum & 3.34 & 9 & 24 & 37.6 & 0.129 & 24,938 & 7.5 & 919 & 60.8 & 0.698 \\
\hline Platypus $\ddagger$ & 0.41 & 26 & NA & 43.3 & 0.296 & 14,686 & 35.9 & 929 & 56.8 & 0.785 \\
\hline
\end{tabular}

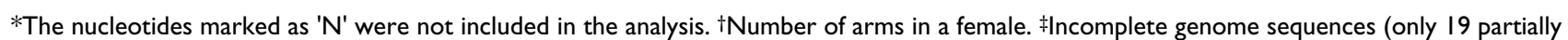
assembled chromosomes). NA, not available.

of CGIs per Mb) vary greatly among genomes. The dog genome has the largest number of CGIs $(58,327)$ and the platypus genome has the highest CGI density (35.9 CGIs/Mb). Remarkably, the number of CGIs in the dog genome is nearly three times that in the rat $(19,568)$ or mouse $(20,458)$ genome, even though the number of dog genes has been estimated to be smaller than those of human or mouse genes (dog, 19,300 [18]; human, 20,000-25,000 [19]; mouse, approximately 30,00o [11]). The CGI density (per Mb) ranges from 7.5 (opossum) to 35.9 (platypus) in the 10 genomes investigated. These results suggest that, although genes are often associated with CGIs, the extant CGIs are distributed very differently among genomic regions (for example, genes versus non-coding regions) in mammalian genomes.

\section{Correlations between CGI density and other genomic features}

We examined the correlations between CGI density and other genomic features. Because of incomplete genome sequence and lack of some chromosome data in platypus, we present the correlation results only for the other nine genomes; the conclusion will likely be the same when the platypus data become available (Additional data file 2). We found a highly significant positive correlation between CGI density and number of chromosome pairs in a genome $(r=0.88, P=7.9$ $\times 10^{-4}$; Figure 1a) and a significant correlation between CGI density and number of chromosome arms $(r=0.62, P=$ o.037). As expected, there was a significant positive correlation between CGI density and $\mathrm{Obs}_{\mathrm{CpG}} / \operatorname{Exp}_{\mathrm{CpG}}(r=0.63, P=$ o.035). No significant correlation was found between CGI density and genome size $(r=-0.53, P=0.073)$ or genome GC content $(r=0.24, P=0.27)$.

There were a total of 219 chromosomes available in these 9 genomes after excluding the $\mathrm{Y}$ chromosomes. We found a highly significant negative correlation between CGI density and $\log _{10}$ (chromosome size) $\left(r=-0.51, P=2.6 \times 10^{-16}\right.$; Figure 1b), a highly significant positive correlation between CGI density and GC content of the chromosome ( $r=0.65, P=3.5 \times$ $10^{-28}$; Figure 1c), and a highly significant positive correlation between CGI density and $\mathrm{Obs}_{\mathrm{CpG}} / \operatorname{Exp}_{\mathrm{CpG}}(r=0.75, P=2.8 \times$ 10-41; Figure 1d). We further separated the chromosomes into different groups by their sizes $(<25,25-50,50-75,75-100$, 100-150, 150-200, and >200 Mb). Interestingly, as the average size of a chromosome group increases, the CGI density decreases (Table 2). Indeed, the CGI density in small mammalian chromosomes ( three times that in large chromosomes (size $>200 \mathrm{Mb}$ ). We noted that the platypus $(2 n=52)$, which has six pairs of large chromosomes but many small chromosomes [20], has a much higher CGI density than the other nine mammalian genomes (Table 1). These results are consistent with the previous observation that CGIs are highly concentrated on the microchromosomes in chickens [21].

The dog has overall smaller chromosomes and high CGI density, while the opossum has a few large chromosomes and low CGI density. To check whether our correlation analysis was largely driven by these two species, we performed a similar analysis but excluded the dog and opossum data. The same conclusion still held. For example, we found a significant correlation between CGI density and number of chromosome pairs ( $r=0.75, P=0.026)$ and a significant correlation between CGI density and $\log _{10}$ (chromosome size) $(r=-0.49$, $\left.P=5.9 \times 10^{-12}\right)$.

CGIs are considered gene markers, so they are expected to highly correlate with gene density $[2,22]$. It is interesting to investigate whether the above correlation results still hold when gene information is excluded. We identified CGIs in the 


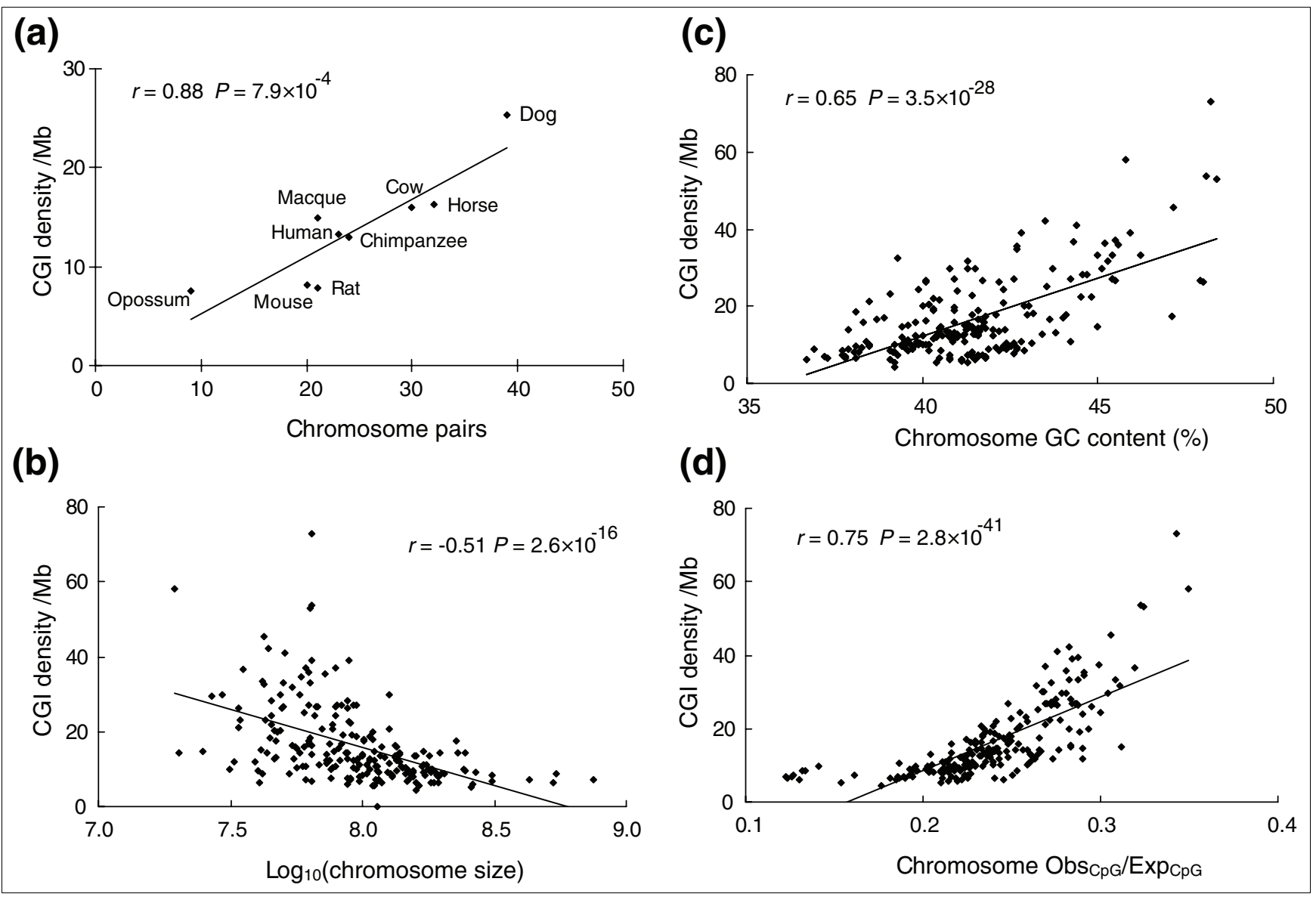

Figure I

Correlations between CGI density and genomic features in nine mammalian genomes. The platypus chromosomes were excluded because of incomplete genome sequence data and chromosome data. (a) CGI density (per Mb) versus number of chromosome pairs. (b) CGI density (per Mb) versus $\log _{10}$ (chromosome size). The $Y$ chromosomes were excluded because of insufficient data. (c) CGI density (per Mb) versus chromosome GC content (\%). (d) CGI density (per Mb) versus chromosome Obs ${ }_{\mathrm{CPG}_{\mathrm{p}}} /$ ExP $_{\mathrm{CP}_{\mathrm{p}} \text {. }}$.

intergenic regions of nine mammalian genomes and found significant correlations between intergenic CGI density and $\log _{10}$ (chromosome size) $\left(r=-0.55, P=7.3 \times 10^{-19}\right)$, GC con- tent of the chromosome $\left(r=0.39, P=8.6 \times 10^{-10}\right)$, and $\mathrm{Obs}_{\mathrm{CpG}} / \operatorname{Exp}_{\mathrm{CpG}}\left(r=0.67, P=3.7 \times 10^{-30}\right)$. Details are shown in Additional data file 3 .

Table 2

\section{CGI densities in chromosomes with different sizes in nine mammalian genomes}

\begin{tabular}{lcc} 
Chromosome size $(\mathrm{Mb})$ & Number of chromosomes & CGI density/Mb \pm SD \\
\hline$<25$ & 5 & $29.7 \pm 17.7$ \\
$25-50$ & 35 & $24.0 \pm 13.2$ \\
$50-75$ & 47 & $21.7 \pm 11.3$ \\
$75-100$ & 43 & $14.7 \pm 7.4$ \\
$100-150$ & 49 & $11.7 \pm 4.6$ \\
$150-200$ & 26 & $9.7 \pm 2.6$ \\
$>200$ & 14 & $9.4 \pm 3.6$ \\
Total & 219 & $16.4 \pm 10.5$
\end{tabular}

$\mathrm{SD}$, standard deviation. 
Table 3

Correlation between CGI density and genomic features in different human genomic regions

\begin{tabular}{|c|c|c|c|c|c|c|c|c|}
\hline & \multicolumn{2}{|c|}{ Gene-associated CGls $(24,228)$} & \multicolumn{2}{|c|}{ Intergenic CGls $(13,026)$} & \multicolumn{2}{|c|}{ Intragenic CGls $(12,136)$} & \multicolumn{2}{|c|}{ TSS CGIs $(11,192)$} \\
\hline & $r$ & $P$ & $r$ & $P$ & $r$ & $P$ & $r$ & $P$ \\
\hline $\log _{10}($ chromosome size) & -0.54 & $3.9 \times 10^{-3}$ & -0.55 & $3.4 \times 10^{-3}$ & -0.55 & $3.1 \times 10^{-3}$ & -0.51 & $7.0 \times 10^{-3}$ \\
\hline GC content & 0.88 & $1.7 \times 10^{-8}$ & 0.87 & $2.9 \times 10^{-8}$ & 0.85 & $1.9 \times 10^{-7}$ & 0.91 & $5.4 \times 10^{-10}$ \\
\hline $\mathrm{Obs}_{\mathrm{CP}_{\mathrm{p}}} / \operatorname{ExP}_{\mathrm{C}_{\mathrm{p}} \mathrm{G}}$ & 0.92 & $1.5 \times 10^{-10}$ & 0.91 & $8.3 \times 10^{-10}$ & 0.92 & $2.5 \times 10^{-10}$ & 0.91 & $1.0 \times 10^{-9}$ \\
\hline
\end{tabular}

It is also interesting to examine whether the correlations between CGI density and other genomic factors would hold in different genomic regions. We used human data because of their high quality annotations. According to gene annotations in the NCBI database, we identified 24,228 CGIs overlapped or within genes (gene-associated CGIs), 13,026 CGIs whose whole sequences were within intergenic regions (intergenic CGIs), 12,136 CGIs whose whole sequences were within gene regions (intragenic CGIs), and 11,192 CGIs overlapped with transcriptional start sites (TSS CGIs) in the human genome. Table 3 shows significant correlations between CGI density and genomic features $\left(\log _{10}\right.$ (chromosome size), GC content, and $\mathrm{Obs}_{\mathrm{CpG}} / \operatorname{Exp}_{\mathrm{CpG}}$ ) in all genomic regions when we compare the data at the chromosome level.

Table 4 summarizes the correlations between CGIs and genomic features based on nine or ten genomes using three CGI identification algorithms.

\section{CGI density and recombination rate}

Recombination rate correlates with both the number of chromosomes and the number of chromosome arms, and elevates the GC content, probably via biased gene conversion [23,24]. Fine-scale recombination rates vary extensively among populations [25,26], genomic regions [27], or the homologous regions between two closely related organisms (human and chimpanzee) $[28,29]$, suggesting a rapid evolution of local pattern of recombination rates. Many genomic features, including $\mathrm{CpG}$ dinucleotide frequencies (but not CGIs or CGI density) in genomic sequences, have been employed to analyze the pattern of recombination rate. Here we examined specifically the relationship between CGI density and recombination rate at the genome level. We retrieved human recombination rate data (window size, $1 \mathrm{Mb}, 2,772$ windows) from the UCSC Genome Browser [30]. We found a significant positive correlation between CGI density and recombination rate $\left(r=0.18, P=1.1 \times 10^{-22}\right)$.

We obtained another set of recombination rate data (in $5 \mathrm{Mb}$ and $10 \mathrm{Mb}$ windows) for the human, mouse and rat from Jensen-Seaman et al. [31]. We discarded those regions that had more than $50 \%$ 'N's ('N' denotes an uncertain nucleotide in the sequence) or whose recombination rate was 0 . In the latter case, it was likely due to insufficient available genetic markers or a small number of meioses used to construct the genetic maps [31]. Again, we found a significant correlation between CGI density and recombination rate, regardless of window size ( $5 \mathrm{Mb}$ or $10 \mathrm{Mb}$; Table 5 and Additional data file 4). For example, the correlation coefficient was $0.33(P=5.9$ $\times 10^{-16}$ ) for human recombination rates measured in a $5 \mathrm{Mb}$ window (Figure 2). The correlation became stronger as the window size increased. Furthermore, the extent of the correlation was different among the three genomes. For example, the coefficients were 0.33 (human), 0.24 (mouse), and 0.17 (rat), respectively, when the $5 \mathrm{Mb}$ window was used.

Recombination rates were found to increase from the centromeric towards telomeric regions [31]. Interestingly, we observed a trend of higher CGI density in the telomeric regions (Figure 3) in many chromosomes. This feature supports a positive correlation between CGI density and recombination rate. However, this finding is opposite to a previous observation of no correlation between CGI features and chromosomal telomere position based on a small gene dataset [17].

\section{Comparison of CGIs in non-mammalian vertebrate genomes}

To retrieve information on the CGIs in vertebrate genomes, we scanned CGIs in seven non-mammalian vertebrate genomes, including the chicken, lizard and five fish (tetraodon, medaka, zebrafish, stickleback and fugu) genomes. Except for lizard and fugu, all these genomes had assembled chromosomes.

Table 6 shows the CGIs and other genome information for the seven non-mammalian vertebrates. The CGI density had a much wider range (14.7-161.6 per $\mathrm{Mb}$ ) among these genomes. The CGI densities in the chicken (23.0 per $\mathrm{Mb}$ ) and green anole lizard (25.9 per $\mathrm{Mb}$ ) were similar to that in the dog (25.3 per $\mathrm{Mb}$ ), higher than that in the other eight therians, but lower than that (35.9 per $\mathrm{Mb}$ ) in the platypus (prototherian) (Table 1). It is worth noting that both the chicken and platypus have many small chromosomes. The chicken karyotype consists of 39 chromosomes, of which 33 are classified as microchromosomes [32]. At the DNA sequence level, chicken chromosomes were separated into three groups (large macrochromosomes, intermediate chromosomes and microchro- 
Table 4

\begin{tabular}{|c|c|c|c|c|}
\hline Algorithm & Genomic features & $r$ & $P$ & Shown in figure \\
\hline \multirow[t]{8}{*}{ TJ (9 genomes) } & Chromosome pairs & 0.88 & $7.9 \times 10^{-4}$ & la \\
\hline & $\log _{10}($ chromosome size) & -0.51 & $2.6 \times 10^{-16}$ & $\mathrm{Ib}$ \\
\hline & Chromosome GC content & 0.65 & $3.5 \times 10^{-28}$ & Ic \\
\hline & Chromosome $\mathrm{Obs}_{\mathrm{CPG}_{\mathrm{p}}} / \operatorname{ExP}_{\mathrm{Cp}_{\mathrm{p}}}$ & 0.75 & $2.8 \times 10^{-41}$ & Id \\
\hline & Chromosome arms & 0.62 & 0.037 & \\
\hline & Genome size & -0.53 & $0.073^{*}$ & \\
\hline & Genomic GC content & 0.24 & $0.27^{*}$ & \\
\hline & Genomic Obs ${ }_{C p G} / \operatorname{ExP}_{C p G}$ & 0.63 & 0.035 & \\
\hline \multirow[t]{4}{*}{ TJ (9 genomes, intergenic CGIs) } & Chromosome pairs & 0.79 & 0.005 & S2a \\
\hline & $\log _{10}($ chromosome size $)$ & -0.55 & $7.3 \times 10^{-19}$ & $S 2 b$ \\
\hline & Chromosome GC content & 0.39 & $8.6 \times 10^{-10}$ & $S 2 c$ \\
\hline & Chromosome Obs ${ }_{\mathrm{CPG}} / \operatorname{ExP}_{\mathrm{Cp}}$ & 0.67 & $3.7 \times 10^{-30}$ & S2d \\
\hline \multirow[t]{4}{*}{ TJ (10 genomes) } & Chromosome pairs & 0.58 & 0.039 & Sla \\
\hline & $\log _{10}($ chromosome size) & -0.70 & $2.6 \times 10^{-37}$ & SIb \\
\hline & Chromosome GC content & 0.64 & $3.7 \times 10^{-29}$ & SIc \\
\hline & Chromosome Obs ${ }_{C p G} / \operatorname{Exp}_{C_{p} G}$ & 0.89 & $1.5 \times 10^{-81}$ & SId \\
\hline \multirow[t]{4}{*}{ GF (9 genomes) } & Chromosome pairs & 0.92 & $2.0 \times 10^{-4}$ & S5a \\
\hline & $\log _{10}($ chromosome size $)$ & -0.63 & $1.3 \times 10^{-25}$ & S5b \\
\hline & Chromosome GC content & 0.72 & $3.2 \times 10^{-37}$ & S5c \\
\hline & Chromosome Obs ${ }_{C p G} / \operatorname{Exp}_{C p G}$ & 0.81 & $2.4 \times 10^{-53}$ & S5d \\
\hline \multirow[t]{4}{*}{ CpGcluster ( 9 genomes) } & Chromosome pairs & 0.81 & 0.004 & S6a \\
\hline & $\log _{10}($ chromosome size $)$ & -0.52 & $1.6 \times 10^{-16}$ & S6b \\
\hline & Chromosome GC content & 0.21 & 0.001 & S6c \\
\hline & Chromosome $\mathrm{Obs}_{\mathrm{Cp}} / \operatorname{ExP}_{\mathrm{Cp}_{\mathrm{p}}}$ & 0.61 & $5.5 \times 10^{-24}$ & S6d \\
\hline
\end{tabular}

*Insignificant correlation. GF, Gardiner-Garden and Frommer's algorithm; TJ, Takai and Jones' algorithm. mosomes) by the International Chicken Genome Sequencing Consortium [33]. Using this classification, we found that CGI density in the 20 chicken microchromosomes (51.7 per $\mathrm{Mb}$ ) was much higher than that $(15.0$ per $\mathrm{Mb})$ in the 6 large macrochromosomes (Table 6), consistent with an earlier report [21]. We did not estimate the CGI density in the large or small chromosomes of platypus because the available assembled genome sequences $(410 \mathrm{Mb})$ represent only a small portion of the genome, which is expected to be about the same size as the human genome [20].

CGI densities in the five fish genomes varied to a much greater extent than in the mammalian genomes. The CGI densities in tetraodon (161.6 per Mb) and stickleback (157.8 per $\mathrm{Mb}$ ) were about 11 times that in zebrafish (14.7 per Mb). The $\mathrm{Obs}_{\mathrm{CpG}} / \operatorname{Exp}_{\mathrm{CpG}}$ ratios in the fish genomes (0.479-0.662) were also much higher than those (0.129-0.296) in the mammalian, the chicken (0.248) and the lizard (0.296) genomes. Fishes are cold-blooded vertebrates and lack GC-rich isochores [34]. An early study found certain fish did not have elevated GC content in nonmethylated CGIs [35], so our comparison of CGIs in fishes should be taken with caution.

In contrast to the observation in mammalian genomes, the correlation between CGI density and number of chromosome pairs in the seven non-mammals was not significant $(r=-$ $0.42, P=0.17)$. We further examined CGI density at the chromosome level in the five non-mammalian genomes (chicken, tetraodon, stickleback, medaka and zebrafish), whose assembled chromosomes are available, and compared it to the nine mammalian genomes. To distinguish the features of CGIs among different genomes, we separated them into different groups: primates (human, chimpanzee and macaque), rodents (mouse and rat), dog-horse-cow, opossum, chicken and fish (tetraodon, stickleback, medaka and zebrafish). Figure 4 shows the plots of CGI density over chromosome GC content. Although there is an overall trend of increasing CGI density with chromosome GC content in both the mammals and non-mammals, their distributions of CGI densities over the chromosome GC content are different. In mammals, CGI 
Table 5

\begin{tabular}{|c|c|c|c|}
\hline & Window size (Mb) & $r$ & $P$ \\
\hline \multirow[t]{3}{*}{ Human } & I & 0.18 & $1.1 \times 10^{-22}$ \\
\hline & 5 & 0.33 & $5.9 \times 10^{-16}$ \\
\hline & 10 & 0.40 & $1.7 \times 10^{-12}$ \\
\hline \multirow[t]{2}{*}{ Mouse } & 5 & 0.24 & $3.6 \times 10^{-7}$ \\
\hline & 10 & 0.33 & $8.0 \times 10^{-8}$ \\
\hline \multirow[t]{2}{*}{ Rat } & 5 & 0.17 & $8.1 \times 10^{-5}$ \\
\hline & 10 & 0.26 & $1.7 \times 10^{-5}$ \\
\hline
\end{tabular}

The detailed distributions are shown in Additional data file 4. Human recombination rate data measured with a I Mb window were based on the deCODE genetic map and downloaded from the UCSC Genome Browser [30]. Recombination rate data measured with $5 \mathrm{Mb}$ and 10 $\mathrm{Mb}$ windows were prepared by Jensen-Seaman et al. [3I] and downloaded from the associated supplementary material website.

density is high in dog-horse-cow and low in rodents, but extensive overlaps are seen among different groups, especially between primates and other groups (Figure 4a). This pattern is more evident in the plots of CGI density versus $\log _{10}$ (chromosome size) or versus chromosome $\mathrm{Obs}_{\mathrm{CpG}} /$ $\operatorname{Exp}_{\mathrm{CpG}}$ ratios (Additional data file 5). Interestingly, we found an overall distinct distribution pattern among non-mammal genomes, especially among the fish genomes (Figure 4b). The chromosomes from each fish genome clustered but they were separated from other fish genomes (Figure 4b, Additional data file 5). Finally, when all species were plotted together, there were overlaps between mammals and non-mammals, but overall, fish chromosomes and chicken microchromosomes could be separated from the mammalian chromosomes (Figure 4b, Additional data file 5).

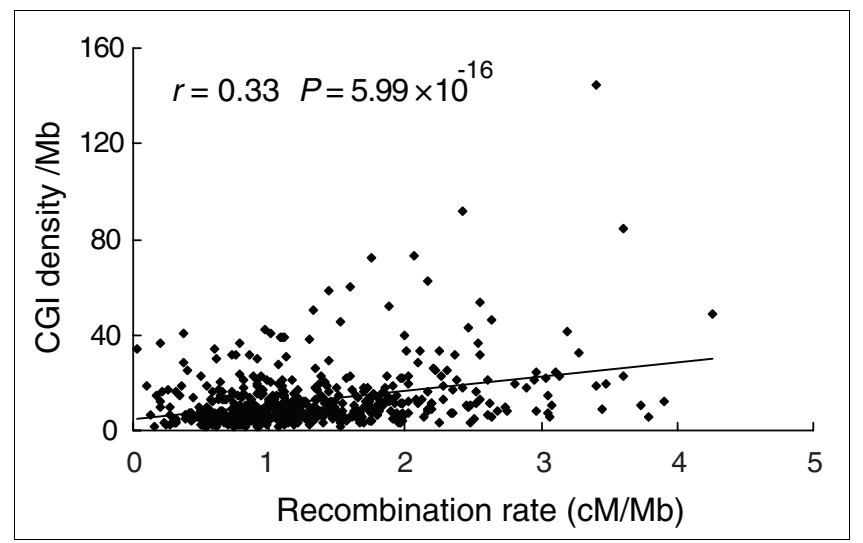

Figure 2

Correlation between CGI density and recombination rate $(\mathrm{cM} / \mathrm{Mb})$ in the human genome; a $5 \mathrm{Mb}$ window was used.

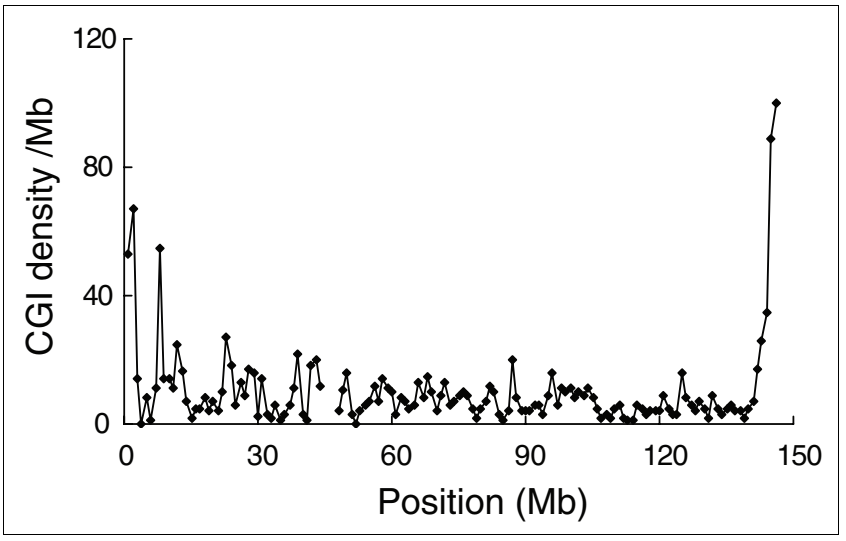

Figure 3

Distribution of CGI density (per Mb) on human chromosome 8 . The data indicate a trend of higher CGI density in telomeric regions.

\section{Discussion \\ Influence of CGI identification algorithms}

There are three major algorithms for identifying CGIs in a genomic sequence (reviewed in the Background). The major aim in this study is to investigate and compare the CGIs in today's mammalian genomes, rather than to identify CGIs in the mammalian ancestral sequences. Thus, our analysis may provide insights into how CGIs have evolved and their association with gene function and other genomic factors. Since CGIs have been widely documented to be approximately $1 \mathrm{~kb}$ long $[2,6]$, Takai and Jones' stringent criteria seem to be the most appropriate for our analysis. To assure the reliability of our analysis, we performed similar analysis using GardinerGarden and Frommer's algorithm (only on the non-repeat portions of the genomes) and CpGcluster with the ten mammalian genomes and seven other vertebrate genomes under study. The conclusions were the same; see detailed results in Table 4 and Additional data files 6 and 7 . For example, there was a significant positive correlation between CGI density and chromosome number, using Gardiner-Garden and Frommer's algorithm $\left(r=0.92, P=2.0 \times 10^{-4}\right.$; Additional data file 6) or CpGcluster ( $r=0.81, P=0.004$; Additional data file 7 ).

However, we found that the number of CGIs identified by CpGcluster or Gardiner-Garden and Frommer's algorithm was remarkably larger than that identified by Takai and Jones' algorithm (Additional data file 8); for example, the numbers of CGIs identified in the human genome was 37,531 (Takai and Jones), 76,678 (Gardiner-Garden and Frommer), and 197,727 (CpGcluster). The number of genes was estimated to be approximately in the range 20,000-30,000 in mammalian genomes (Additional data file 1). Since CGIs have been widely considered as gene markers, both the GardinerGarden and Frommer algorithm and CpGcluster likely identified either many CGIs that are not associated with genes or multiple CGIs that share one gene. To address the latter case, we evaluated the length distribution of CGIs identified by the three algorithms. Among all these vertebrate genomes, the 
Table 6

\begin{tabular}{|c|c|c|c|c|c|c|c|c|c|}
\hline \multirow[b]{2}{*}{ Species } & \multicolumn{4}{|c|}{ Genome } & \multicolumn{5}{|c|}{ CPG islands } \\
\hline & $\begin{array}{l}\text { Length } \\
(\mathrm{Mb})^{*}\end{array}$ & $\begin{array}{c}\text { Number of } \\
\text { chromosome } \\
\text { pairs }\end{array}$ & $\begin{array}{c}\text { GC } \\
\text { content } \\
(\%)\end{array}$ & $\begin{array}{l}\text { Obs }_{C_{p} G} \\
\operatorname{Exp}_{C_{p} G}\end{array}$ & $\begin{array}{c}\text { Number of } \\
\text { CGls }\end{array}$ & $\begin{array}{l}\text { CGI density } \\
\text { (/Mb) }\end{array}$ & $\begin{array}{l}\text { Avgerage } \\
\text { length (bp) }\end{array}$ & $\begin{array}{c}\mathrm{GC} \\
\text { content } \\
(\%)\end{array}$ & $\begin{array}{l}\operatorname{Obs}_{C_{p G}} / \\
\operatorname{ExP}_{C_{p} G}\end{array}$ \\
\hline Chickent & 985 & 39 & 41.4 & 0.248 & 22,623 & 23.0 & 1,098 & 60.0 & 0.844 \\
\hline Microchromosome & 167 & 20 & 45.7 & 0.305 & 8,634 & 51.7 & 1,040 & 60.4 & 0.810 \\
\hline Macrochromosome & 674 & 6 & 40.0 & 0.219 & 10,125 & 15.0 & 1,138 & 59.6 & 0.863 \\
\hline Lizard & $\mathrm{I}, 742$ & 18 & 40.4 & 0.296 & $45,|7|$ & 25.9 & 899 & 56.8 & 0.728 \\
\hline Tetraodon & 187 & 21 & 45.9 & 0.601 & 30,175 & 161.6 & 1,013 & 56.7 & 0.782 \\
\hline Stickleback & 391 & 21 & 44.5 & 0.662 & 61,768 & 157.8 & 824 & 55.8 & 0.842 \\
\hline Medaka & 582 & 24 & 40.1 & 0.479 & 21,522 & 37.0 & 746 & 55.8 & 0.784 \\
\hline Zebrafish & 1,524 & 25 & 36.5 & 0.531 & 22,392 & 14.7 & 1,162 & 57.0 & 0.869 \\
\hline Fugu & 351 & 22 & 45.5 & 0.565 & 47,251 & 134.5 & 872 & 56.0 & 0.808 \\
\hline
\end{tabular}

*The nucleotides marked as ' $N$ ' were not included in the analysis. tOnly 30 chromosomes were used in the analysis because chromosomes $29-3 \mathrm{I}$ and 33-38 were too small to assemble [39]. The microchromosomes included chromosomes GGAII-28, 32 and W and the macrochromosomes included chromosomes GGAI-5 and Z.

majority of CGIs identified by CpGcluster were shorter than $500 \mathrm{bp}$ (Additional data file 8), which is the minimum length in Takai and Jones' algorithm. For example, the proportions of human CGIs identified by CpGcluster were 44.3\% (<200 bp), 45.9\% (200-500 bp), 7.3\% (500-1,00o bp), 1.9\% (1,000$1,500 \mathrm{bp}), 0.4 \%$ (1,500-2,00o bp), and $0.2 \%$ ( $\geq 2,000 \mathrm{bp})$. For Gardiner-Garden and Frommer's algorithm, the proportion of CGIs shorter than 500 bp was also large, for example, $65.8 \%$ in the human CGIs and $64.8 \%$ in the opossum CGIs (Additional data file 8). Based on the evaluation above, we consider that our analysis using Takai and Jones' algorithm is the most reliable and appropriate, though further evaluation of species-specific algorithms may enhance our results.

\section{Evolution of CGIs}

It was hypothesized that CGIs arose once at the dawn of vertebrate evolution and vertebrate ancestral genes were embedded in entirely non-methylated DNA during the divergence of vertebrates [9]. Genome-wide methylation has been found to be common in vertebrates (except for promoter-associated CGIs) and fractional methylation common in invertebrates. The transition from fractional to global methylation likely occurred around the origin of vertebrates [36]. Many CGIs might have lost their typical features due to de novo methylation at their $\mathrm{CpG}$ sites and subsequent high deamination rates at the newly methylated $\mathrm{CpG}$ sites, leading to TpG and $\mathrm{CpA}$ dinucleotides. Excess of TpGs and CpAs as well as other vanishing CGI features (decreasing length, $\mathrm{Obs}_{\mathrm{CpG}} / \operatorname{Exp}_{\mathrm{CpG}}$ ratio and GC content) has been found in the homologous gene regions, evidence of frequent CGI losses in mouse and human genes and a faster loss rate in mice $[7,9,17]$. Recent methylation studies revealed weak CGIs in promoter regions (promoters with intermediate CpG content, ICPs), most of which were not found in the CGI library, had a faster loss rate of CpGs than stronger CGIs (promoters with high CpG content,
HCPs), suggesting that strong CGIs might be protected from methylation and are thus better conserved during evolution $[22,37,38]$. Using the data in Weber et al. [37] and Mikkelsen et al. [38], we found that HCP density has stronger correlations with genomic features than ICPs in both the human and mouse genomes. The CGIs identified by the Takai-Jones algorithm are different from HCPs or ICPs. However, when we separated the promoter-associated CGIs identified by the Takai-Jones algorithm into HCGIs (those that satisfied the HCP criteria) and non-HCGIs, we also found that HCGIs had stronger correlations with genomic features than non-HCGIs. This supports the observations from the methylation studies mentioned above. Although loss of CGIs is likely a major evolutionary scenario in mammals, little comparative analysis at the DNA sequence level has been performed yet, because CGIs have been thought to be poorly conserved between species $[7,9]$. Our CGI analysis indicated that rodents have the lowest CGI density and most other eutherians have moderate CGI density when compared to platypus (Table 1). Platypus is one of the only three extant monotremes and has a fascinating mixture of features typical of mammals and of reptiles and birds. Monotremes (mammalian subclass Prototheria) are the oldest branch of the mammalian tree, diverging 210 million years ago from the therian mammals [20]. Although the platypus genome is incomplete, its higher CGI density is likely true because high frequencies of GC and CG dinucleotides and high GC content have been reported [20]. Further, our analysis of the chicken (bird) and green anole lizard genomic sequences, the only reptilian genome available at present, showed higher CGI density than most of the therians (except dogs) we examined. These data support an overall decrease in CGIs in mammalian genomes.

Below we discuss specific CGI features of a few species. The low number of CGIs in the rodent genome is likely due to a 


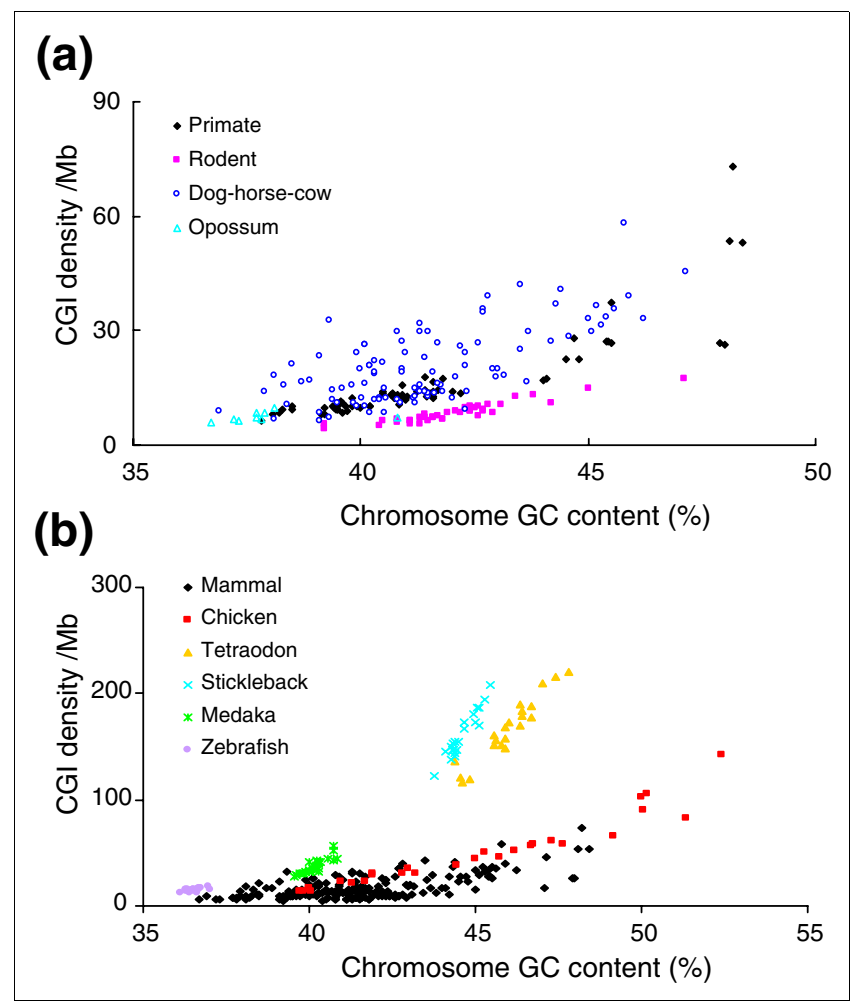

Figure 4

CGI density comparison between mammals and non-mammals. This figure shows the distribution of CGI density (per Mb) versus chromosome GC content (\%). (a) Comparison of four groups in mammals. (b) Comparison of mammals, chicken and fish.

much higher rate of CGI loss and a weaker selective constraint in the rodent lineage $[7,17]$. Interestingly, the dog has a notably large number of CGIs and high CGI density among the nine therians investigated. Our further analysis revealed that the difference is due to the substantial enrichment of CGIs in dog's intergenic and intronic regions, while the number of CGIs associated with the $5^{\prime}$ end of genes is similar to the human and the mouse (data not shown). Whether and how CGIs have accumulated in dog requires further investigation. It is also worth noting that opossum, which belongs to metatheria, is another evolutionarily ancient lineage of mammals. The CGI density is very low (7.5 per Mb). This is likely attributed to its large chromosomes (Table 1), as large chromosomes are correlated with low CGI density (Figure 1). Large chromosomes reduce recombination rate, which has a positive correlation with CGI density (Figure 2).

Other possible factors that might influence CGI density It is interesting to examine whether species traits such as lifespan, body temperature and body mass are related to CGI density. The small body size and short lifespan of mice were speculated to allow for their tolerance towards leaky control of gene activity, including erosion of CGIs [17]. A previous study also revealed that methylation status is correlated with body temperatures in fish and affected by the local environ-

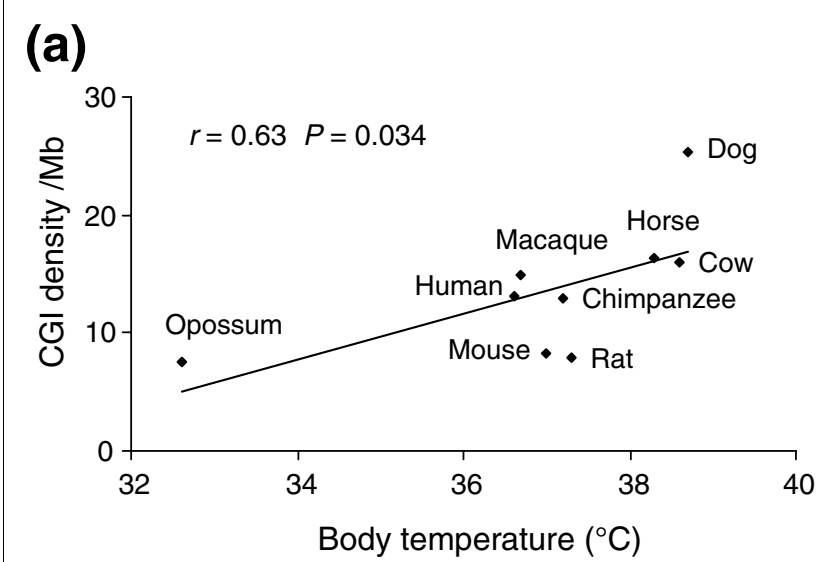

(b)

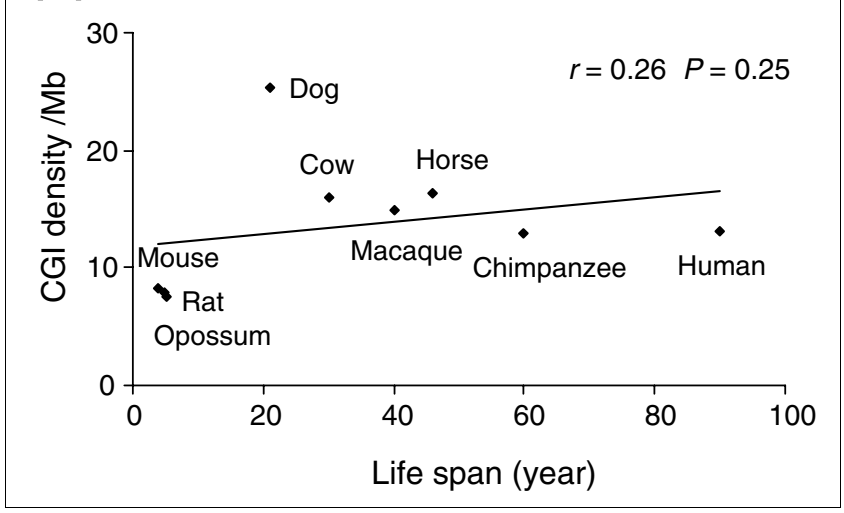

Figure 5

Correlation between CGI density and other genetic factors. (a) Significant correlation between CGI density and body temperature. (b) Insignificant correlation between CGI density and lifespan.

ment [39]. It was also proposed that GC content of the isochores is driven by increasing body temperature, which has selective advantages because of being more thermally stable in higher GC-content regions [40]. Our correlation analysis found a significant correlation between CGI density and body temperature in eight eutherians $(r=0.67, P=0.035)$ and nine therians ( $r=0.63, P=0.034$; Figure $5 \mathrm{a}$ ). However, when platypus and/or chicken were added, the correlation became insignificant. Furthermore, we did not find a significant correlation between CGI density and lifespan in the eight eutherians $(r=0.14, P=0.38)$ or nine therians $(r=0.26, P=0.25$; Figure $5 \mathrm{~b})$. Some factors might have affected the estimation of lifespan, making the analysis unreliable. First, living environments are much different between domesticated and wild animals; meanwhile, modern medical treatment has increased human longevity. Second, lifespan in the same species may differ according to factors such as sex [41] and hormonal regulation $[42,43]$. Third, the divergence among mammals is low when compared to other vertebrates. In summary, our analysis of these species traits should be considered preliminary. 
Table 7

\begin{tabular}{|c|c|c|c|}
\hline Common name & Species name & Sequence build & Data source \\
\hline \multicolumn{4}{|l|}{ Mammal } \\
\hline Human & Homo sapiens & 35.1 & $\mathrm{NCBI}[44]$ \\
\hline Chimpanzee & Pan troglodytes & 2.1 & $\mathrm{NCBI}[44]$ \\
\hline Macaque & Macaca mulatta & I.I & $\mathrm{NCBI}[44]$ \\
\hline Mouse & Mus musculus & 34.1 & $\mathrm{NCBI}[44]$ \\
\hline Rat & Rattus norvegicus & 4.1 & $\mathrm{NCBI}[44]$ \\
\hline Dog & Canis familiaris & 2.1 & $\mathrm{NCBI}[44]$ \\
\hline Cow & Bos taurus & 3.1 & $\mathrm{NCBI}[44]$ \\
\hline Horse & Equus caballus & I.I & $\mathrm{NCBI}[44]$ \\
\hline Opossum & Monodelphis domestica & 2.1 & $\mathrm{NCBI}[44]$ \\
\hline Platypus* & Ornithorhynchus anatinus & I.I & $\mathrm{NCBI}[44]$ \\
\hline \multicolumn{4}{|l|}{ Non-mammal vertebrate } \\
\hline Chickent & Gallus gallus & 2.1 & $\mathrm{NCBI}[44]$ \\
\hline Green anole lizard $\ddagger$ & Anolis carolinensis & anoCarl & UCSC [30] \\
\hline Tetraodon & Tetraodon nigroviridis & tetNig I & UCSC [30] \\
\hline Stickleback & Gasterosteus aculeatus & gasAcul & UCSC [30] \\
\hline Medaka & Oryzias latipes & oryLat I & UCSC [30] \\
\hline Zebrafish & Danio rerio & danRer5 & UCSC [30] \\
\hline Fugu $\ddagger$ & Takifugu rubripes & fr2 & UCSC [30] \\
\hline
\end{tabular}

*The platypus genome was partially assembled. Only chromosomes I-7, 10-12, 14, 15, 17, 18, 20, XI-X3, and X5 were available. tOnly chromosomes I-28, 32, W, and Z were available. $¥$ No assembled chromosomes.

\section{Conclusion}

This study represents a systematic comparative genomic analysis of CGIs and CGI density at the DNA sequence level in mammals. It reveals significant correlations between CGI density and genomic features such as number of chromosome pairs, chromosome size, and recombination rate. Our results suggest a genome evolution scenario in which an increase in chromosome number increases the rate of recombination, which in turn elevates GC content to help prevent loss of CGIs and maintain CGI density. We compared CGI features in other non-mammalian vertebrates and discussed other factors such as body temperature and lifespan that have previously been speculated to influence sequence composition evolution.

\section{Materials and methods}

\section{Genome sequences and genome information}

We downloaded the assembled genome sequences (ten mammalian genomes and seven non-mammalian vertebrate genomes) from the National Center for Biotechnology Information (NCBI) [44] and the UCSC Genome Browser [30]. The species names and data sources are provided in Table 7 . The repeat-masked sequences of these genomes were downloaded from the UCSC Genome Browser [30]. We used the EMBOSS package [45] to calculate the genome size, the GC content and the $\mathrm{Obs}_{\mathrm{CpG}} / \operatorname{Exp}_{\mathrm{CpG}}$ ratios. Gene numbers were based on the annotations in Ensembl [46] and also in the literature (details are shown in Additional data file 1). At present, it remains a great challenge to obtain an accurate estimation of the gene number in a genome, but we suspect that the actual gene numbers in these genomes are likely in a smaller range than the range 20,000-30,000 in Additional data file 1.

\section{Identification of CpG islands}

We used three algorithms to identify CGIs. First, we used the stringent search criteria in the Takai and Jones algorithm [14]: GC content $\geq 55 \%, \mathrm{Obs}_{\mathrm{CpG}} / \operatorname{Exp}_{\mathrm{CpG}} \geq 0.65$, and length $\geq 500 \mathrm{bp}$. Second, we used the algorithm originally developed by Gardiner-Garden and Frommer [13]: GC content $>50 \%$, $\mathrm{Obs}_{\mathrm{CpG}} / \operatorname{Exp}_{\mathrm{CpG}}>0.60$, and length $>200 \mathrm{bp}$. Because some repeats (for example, Alu) meet these criteria, we scanned CGIs in the non-repeat portions of these genomes only, as similarly done in other genome-wide identification studies $[2,11]$. For both the Takai and Jones and the Gardiner-Garden and Frommer algorithms, we used the $\mathrm{CpG}$ island searcher program (CpGi130) available at [47]. Third, we used CpGclus- 
ter developed by Hackenberg et al. [15] to scan CGIs in the whole genome.

We used the method of Jiang and Zhao [48] to identify CGIs in different genomic regions (genes, intergenic regions, intragenic regions, and TSS regions). Briefly, we compared the locations of CGIs with the coordinates of genic, intergenic, and intragenic regions and TSSs based on the human gene annotation information from the NCBI database (build 35.1) $[44,49]$. CGIs overlapped with any genes were classified as gene-associated CGIs; CGIs whose whole sequences were in intergenic regions were classified as intergenic CGIs; CGIs whose sequences were in gene regions were classified as intragenic CGIs; and CGIs overlapped with TSSs were classified as TSS CGIs.

\section{Recombination rate and CGI density}

We retrieved human recombination rate data based on the deCODE genetic map [50] from the UCSC Genome Browser [30]. The recombination rates were measured in $1 \mathrm{Mb}$ windows. We obtained another set of recombination rates from Jensen-Seaman et al. [31]. These data were measured in $5 \mathrm{Mb}$ and $10 \mathrm{Mb}$ windows for the human, mouse and rat and are available in the supplementary material for Jensen-Seaman et al. [31]. For both datasets, we discarded those regions having more than $50 \%$ 'N's [31]. We also discarded those regions whose recombination rates were o because of too few genetic markers found in these regions [31].

\section{Body temperature and lifespan in mammals}

Records of body temperature in a species may vary to some extent in the literature because they might be measured in different environments (for example, time of day, season, or geographical location) or different sites of the body. The body temperatures of ten mammals in this study were obtained from the literature (details are shown in Additional data file 9). When a species has a range of body temperatures in the literature, the average was used as the representative temperature. There are several measurements of lifespan, such as maximum lifespan, average lifespan, and lifespan of each sex. We used maximum lifespan, which was based on reports in the literature and from the AnAge database [51] (Additional data file 9).

\section{Abbreviations}

CGI, CpG island; HCGI, CGI satisfying the HCP criteria; HCP, high $\mathrm{CpG}$ content promoter; ICP, intermediate CpG content promoter; TSS, transcriptional start site.

\section{Authors' contributions}

LH prepared the data, carried out the data analysis, and contributed to the writing of the manuscript. BS participated in study design and coordination. WHL participated in study design and contributed to the writing of the manuscript. ZZ conceived of the study, participated in the data analysis and interpretation, and contributed to the writing of the manuscript. All authors read and approved the final manuscript.

\section{Additional data files}

The following additional data are available. Additional data file 1 is a table that lists the numbers of genes estimated in mammalian genomes. Additional data file 2 shows the correlations between CGI density and genomic features in ten mammalian genomes (including platypus). Additional data file 3 shows the correlations between intergenic CGI density and genomic features in nine mammalian genomes. Additional data file 4 shows the correlations between CGI density and average recombination rate $(\mathrm{cM} / \mathrm{Mb})$ in the human, mouse and rat genomes. Additional data file 5 provides the comparison of $\mathrm{CpG}$ islands and other genomic features between mammalian and non-mammalian genomes. Additional data file 6 shows the correlations between CGI density and genomic features in mammalian genomes using the Gardiner-Garden and Frommer algorithm in the non-repeat portions of genomes. Additional data file 7 shows the correlations between CGI density and genomic features in mammalian genomes using the CpGcluster algorithm. Additional data file 8 lists the numbers of CGIs in each genome identified by the three algorithms and shows their length distribution. Additional data file 9 lists the body temperature and lifespan for each species.

\section{Acknowledgements}

We thank the two anonymous reviewers for valuable comments. We are grateful to Dr John Speakman for suggestions on estimating lifespan and body temperature. This project was supported by the Thomas $\mathrm{F}$ and Kate Miller Jeffress Memorial Trust Fund and a NARSAD Young Investigator Award to Z Zhao and NIH grants to WH Li.

\section{References}

I. Bird AP: CpG-rich islands and the function of DNA methylation. Nature 1986, 321:209-213.

2. Lander ES, Linton LM, Birren B, Nusbaum C, Zody MC, Baldwin J, Devon K, Dewar K, Doyle M, FitzHugh W, Funke R, Gage D, Harris K, Heaford A, Howland J, Kann L, Lehoczky J, LeVine R, McEwan P, McKernan K, Meldrim J, Mesirov JP, Miranda C, Morris W, Naylor J, Raymond C, Rosetti M, Santos R, Sheridan A, Sougnez C, et al.: Initial sequencing and analysis of the human genome. Nature 200I, 409:860-921.

3. Zhao Z, Zhang F: Sequence context analysis in the mouse genome: Single nucleotide polymorphisms and CPG island sequences. Genomics 2006, 87:68-74.

4. Zhao Z, Zhang F: Sequence context analysis of $\mathbf{8 . 2}$ million single nucleotide polymorphisms in the human genome. Gene 2006, 366:316-324.

5. Bird AP: DNA methylation and the frequency of CpG in animal DNA. Nucleic Acids Res 1980, 8:1499-1504.

6. Antequera F: Structure, function and evolution of $\mathrm{CpG}$ island promoters. Cell Mol Life Sci 2003, 60:1647-1658.

7. Jiang C, Han L, Su B, Li WH, Zhao Z: Features and trend of loss of promoter-associated CpG islands in the human and mouse genomes. Mol Biol Evol 2007, 24:1991-2000.

8. Bird AP: CpG islands as gene markers in the vertebrate nucleus. Trends Genet 1987, 3:342-347.

9. Antequera F, Bird A: Number of CpG islands and genes in human and mouse. Proc Natl Acad Sci USA 1993, 90: I 1995-I 1999. 
10. Venter JC, Adams MD, Myers EW, Li PW, Mural RJ, Sutton GG, Smith HO, Yandell M, Evans CA, Holt RA, Gocayne JD, Amanatides P, Ballew RM, Huson DH, Wortman JR, Zhang Q, Kodira CD, Zheng XH, Chen L, Skupski M, Subramanian G, Thomas PD, Zhang J, Gabor Miklos GL, Nelson C, Broder S, Clark AG, Nadeau J, McKusick VA, Zinder $\mathrm{N}$, et al.: The sequence of the human genome. Science 200|, 29 I: |304-|35|.

II. Mouse Genome Sequencing Consortium, Waterston RH, LindbladToh K, Birney E, Rogers J, Abril JF, Agarwal P, Agarwala R, Ainscough $\mathrm{R}$, Alexandersson M, An P, Antonarakis SE, Attwood J, Baertsch R, Bailey J, Barlow K, Beck S, Berry E, Birren B, Bloom T, Bork P, Botcherby M, Bray N, Brent MR, Brown DG, Brown SD, Bult C, Burton J, Butler J, Campbell RD, Carninci $P$, et al.: Initial sequencing and comparative analysis of the mouse genome. Nature 2002, 420:520-562.

12. Gibbs RA, Weinstock GM, Metzker ML, Muzny DM, Sodergren EJ, Scherer S, Scott G, Steffen D, Worley KC, Burch PE, Okwuonu G, Hines S, Lewis L, DeRamo C, Delgado O, Dugan-Rocha S, Miner G, Morgan M, Hawes A, Gill R, Celera, Holt RA, Adams MD, Amanatides PG, Baden-Tillson H, Barnstead M, Chin S, Evans CA, Ferriera S, Fosler C, et al.: Genome sequence of the Brown Norway rat yields insights into mammalian evolution. Nature 2004, 428:493-521.

13. Gardiner-Garden $M$, Frommer $M$ : CpG islands in vertebrate genomes. J Mol Biol 1987, I 96:261-282.

14. Takai D, Jones PA: Comprehensive analysis of CpG islands in human chromosomes 21 and 22. Proc Natl Acad Sci USA 2002, 99:3740-3745.

15. Hackenberg M, Previti C, Luque-Escamilla PL, Carpena P, MartinezAroza J, Oliver JL: CpGcluster: a distance-based algorithm for CpG-island detection. BMC Bioinformatics 2006, 7:446.

16. Glass JL, Thompson RF, Khulan B, Figueroa ME, Olivier EN, Oakley EJ, Zant GV, Bouhassira EE, Melnick A, Golden A, Fazzari MJ, Greally JM: CG dinucleotide clustering is a species-specific property of the genome. Nucleic Acids Res 2007, 35:6798-6807.

17. Matsuo K, Clay O, Takahashi T, Silke J, Schaffner W: Evidence for erosion of mouse CpG islands during mammalian evolution. Somat Cell Mol Genet 1993, I 9:543-555.

18. Lindblad-Toh K, Wade CM, Mikkelsen TS, Karlsson EK, Jaffe DB, Kamal M, Clamp M, Chang JL, Kulbokas EJ III, Zody MC, Mauceli E, Xie X, Breen M, Wayne RK, Ostrander EA, Ponting CP, Galibert F, Smith DR, Dejong PJ, Kirkness E, Alvarez P, Biagi T, Brockman W, Butler J, Chin CW, Cook A, Cuff J, Daly MJ, DeCaprio D, Gnerre S, et al: Genome sequence, comparative analysis and haplotype structure of the domestic dog. Nature 2005, 438:803-8I9.

19. International Human Genome Sequencing Consortium: Finishing the euchromatic sequence of the human genome. Nature 2004, 431 :931-945.

20. Grutzner F, Graves JA: A platypus' eye view of the mammalian genome. Curr Opin Genet Dev 2004, 14:642-649.

21. McQueen HA, Fantes J, Cross SH, Clark VH, Archibald AL, Bird AP: CpG islands of chicken are concentrated on microchromosomes. Nat Genet 1996, 12:321-324.

22. Illingworth R, Kerr A, Desousa D, Jorgensen H, Ellis P, Stalker J, Jackson D, Clee C, Plumb R, Rogers J, Humphray S, Cox T, Langford C Bird A: A novel CpG island set identifies tissue-specific methylation at developmental gene loci. PLoS Biol 2008, 6:e22.

23. Pardo-Manuel de Villena F, Sapienza C: Recombination is proportional to the number of chromosome arms in mammals. Mamm Genome 2001, I 2:318-322.

24. Meunier J, Duret L: Recombination drives the evolution of GCcontent in the human genome. Mol Biol Evol 2004, 21 :984-990.

25. Evans DM, Cardon LR: A comparison of linkage disequilibrium patterns and estimated population recombination rates across multiple populations. Am J Hum Genet 2005, 76:68I-687.

26. McVean GAT, Myers SR, Hunt S, Deloukas P, Bentley DR, Donnelly $P$ : The fine-scale structure of recombination rate variation in the human genome. Science 2004, 304:58I-584.

27. Myers S, Bottolo L, Freeman C, McVean G, Donnelly P: A fine-scale map of recombination rates and hotspots across the human genome. Science 2005, 3 1 0:32 I-324.

28. Ptak SE, Hinds DA, Koehler K, Nickel B, Patil N, Ballinger DG, Przeworski $M$, Frazer KA, Pääbo S: Fine-scale recombination patterns differ between chimpanzees and humans. Nat Genet 2005, 37:429-434.

29. Winckler W, Myers SR, Richter DJ, Onofrio RC, McDonald GJ, Bontrop RE, McVean GA, Gabriel SB, Reich D, Donnelly P, Altshuler D: Comparison of fine-scale recombination rates in humans and chimpanzees. Science 2005, 308: I07-III.

30. UCSC Genome Browser [http://genome.ucsc.edu/]

31. Jensen-Seaman MI, Furey TS, Payseur BA, Lu Y, Roskin KM, Chen C$F$, Thomas MA, Haussler D, Jacob HJ: Comparative recombination rates in the rat, mouse, and human genomes. Genome Res 2004, I 4:528-538.

32. McQueen HA, Siriaco G, Bird AP: Chicken microchromosomes are hyperacetylated, early replicating, and gene rich. Genome Res 1998, 8:621-630.

33. International Chicken Genome Sequencing Consortium: Sequence and comparative analysis of the chicken genome provide unique perspectives on vertebrate evolution. Nature 2004, 432:695-7I6.

34. Bernardi G, Bernardi G: Compositional transitions in the nuclear genomes of cold-blooded vertebrates. J Mol Evol 1990 , 31:282-293.

35. Cross S, Kovarik P, Schmidtke J, Bird A: Non-methylated islands in fish genomes are GC-poor. Nucleic Acids Res 1991, I9:|469-1474.

36. Tweedie S, Charlton J, Clark V, Bird A: Methylation of genomes and genes at the invertebrate-vertebrate boundary. Mol Cell Biol 1997, I 7:1469-1475.

37. Weber M, Hellmann I, Stadler MB, Ramos L, Paabo S, Rebhan M, Schübeler D: Distribution, silencing potential and evolutionary impact of promoter DNA methylation in the human genome. Nat Genet 2007, 39:457-466.

38. Mikkelsen TS, Ku M, Jaffe DB, Issac B, Lieberman E, Giannoukos G, Alvarez P, Brockman W, Kim TK, Koche RP, Lee W, Mendenhall E, O'Donovan A, Presser A, Russ C, Xie X, Meissner A, Wernig M, Jaenisch R, Nusbaum C, Lander ES, Bernstein BE: Genome-wide maps of chromatin state in pluripotent and lineage-committed cells. Nature 2007, 448:553-560.

39. Varriale A, Bernardi G: DNA methylation and body temperature in fishes. Gene 2006, 385: III-121.

40. Bernardi G: The neoselectionist theory of genome evolution. Proc Natl Acad Sci USA 2007, I 04:8385-8390.

4I. Eskes T, Haanen C: Why do women live longer than men? Eur J Obstet Gynecol Reprod Biol 2007, I33:126-I 33.

42. Brown-Borg HM: Hormonal regulation of aging and life span. Trends Endocrinol Metab 2003, I4:15I-I53.

43. Brown-Borg HM: Hormonal regulation of longevity in mammals. Ageing Res Rev 2007, 6:28-45.

44. NCBI RefSeq Database [ftp://ftp.ncbi.nih.gov/genomes/]

45. Olson SA: EMBOSS opens up sequence analysis. European Molecular Biology Open Software Suite. Brief Bioinform 2002, 3:87-91.

46. Ensembl [http://www.ensembl.org/]

47. CpG Island Searcher Program [http://cpgislands.usc.edu/]

48. Jiang $C$, Zhao $Z$ : Mutational spectrum in the recent human genome inferred by single nucleotide polymorphisms. Genomics 2006, 88:527-534.

49. Zhao Z, Jiang C: Methylation-dependent transition rates are dependent on local sequence lengths and genomic regions. Mol Biol Evol 2007, 24:23-25.

50. Kong A, Gudbjartsson DF, Sainz J, Jonsdottir GM, Gudjonsson SA, Richardsson B, Sigurdardottir S, Barnard J, Hallbeck B, Masson G, Shlien A, Palsson ST, Frigge ML, Thorgeirsson TE, Gulcher JR, Stefansson $\mathrm{K}$ : A high-resolution recombination map of the human genome. Nat Genet 2002, 3 I:241-247.

51. AnAge Database [http://genomics.senescence.info/species/] 